Research Paper

\title{
Identification of Leukocyte telomere length-related genetic variants contributing to predisposition of Esophageal Squamous Cell Carcinoma
}

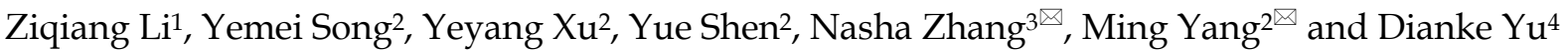 \\ 1. Department of General Surgery, The First Affiliated Hospital of Shandong First Medical University, Jinan, Shandong Province, China. \\ 2. Shandong Provincial Key Laboratory of Radiation Oncology, Cancer Research Center, Shandong Cancer Hospital and Institute, Shandong First Medical \\ University and Shandong Academy of Medical Sciences, Jinan, Shandong Province, China. \\ 3. Department of Radiation Oncology, Shandong Cancer Hospital and Institute, Shandong First Medical University and Shandong Academy of Medical \\ Sciences, Jinan, Shandong Province, China. \\ 4. School of Public Health, Qingdao University, Qingdao, Shandong Province, China.
}

$\square$ Corresponding authors: Ming Yang, Ph.D., Professor, Cancer Research Center, Shandong Cancer Hospital and Institute, Shandong First Medical University and Shandong Academy of Medical Sciences Jinan 250117, Shandong Province, China. Tel \& Fax: 86531-67626536; E-mail: aaryoung@yeah.net; or, Nasha Zhang, M.D., Department of Radiation Oncology, Shandong Cancer Hospital and Institute, Shandong First Medical University and Shandong Academy of Medical Sciences, Jinan 250117, Shandong Province, China. E-mail: wownseva@126.com.

(1) The author(s). This is an open access article distributed under the terms of the Creative Commons Attribution License (https://creativecommons.org/licenses/by/4.0/). See http://ivyspring.com/terms for full terms and conditions.

Received: 2020.02.21; Accepted: 2020.06.04; Published: 2020.06.23

\begin{abstract}
Background: Cancers may arise from cells with dysregulated telomeric functions due to shorten telomere length. We and others previously found that short leukocyte telomere length was associated with markedly evaluated risk of esophageal squamous cell carcinoma (ESCC). Hence, we hypothesized that single nucleotide polymorphisms (SNPs) associated with shorter telomere length may contribute to ESCC predisposition.

Methods: We systematically evaluated association between seven candidate seven SNPs (CXCR4 rs6430612, TERT rs13172201, TERT rs10069690, TERT rs2853676, TERT rs451360, OBFCI rs4387287, and VPS34 rs2162440) and ESCC risk in two case-control sets consisting of 1588 ESCC cases and 1600 controls. Logistic regression models were utilized to estimate associations between SNPs and ESCC susceptibility and odds ratios (ORs) and their $95 \%$ confidence intervals $(95 \% \mathrm{Cls})$ were computed.

Results: We firstly identified three SNPs (rs6430612, rs13172201 and rs4387287) which are significantly associated with telomere length in Chinese populations (all $P<0.05$ ). Importantly, CXCR4 rs6430612 and OBFCI rs4387287 polymorphisms significantly confer reduced risk of ESCC $\left(P=1.7 \times 10^{-7}\right.$ and $\left.P=3.9 \times 10^{-5}\right)$. On the contrary, we observed an evidently increased risk for ESCC development associated with TERT rs 13172201 genetic variant $\left(P=2.2 \times 10^{-4}\right)$.

Conclusions: In summary, rs6430612, rs13172201 and rs4387287 might be key genetic components in complicated regulation of telomere length and contributing to ESCC predisposition. Our results elucidate the prevalent involvement of genetic variants in telomere biology and further provide pathogenic insights into the role of telomeres in cancer development.
\end{abstract}

Key words: telomere; ESCC; CXCR4; TERT; OBFC1

\section{Introduction}

In 2018, GLOBOCAN estimates that there were 572,034 new esophageal cancer cases and 508,585 deaths in the world [1]. Eastern Asia showed the highest region-specific incidence age-standardized rates in both males and females for esophageal cancer (17.9 and 6.8), which are much higher than other regions such as Western Europe (6.8 and 1.7) and
North America (5.5 and 1.5) [1]. In Eastern Asia, the majority of esophageal cancer cases were diagnosed as esophageal squamous cell carcinomas (ESCC) but not esophageal adenocarcinoma [1-3]. There are major risk factors for ESCC, i.e. heavy drinking and smoking as well as dietary components including nutritional deficiencies and nitrosamines [2-4]. However, the full 
etiology of ESCC has yet to be elucidated. In recent years, genome-wide association studies (GWAS) identified a few susceptibility gene loci for ESCC, elucidating that genetic components also contribute to ESCC development [5-14].

Telomeres locating at the ends of linear chromosomes maintain integrity of human genome in cells [15]. Cell divisions lead to inevitable erosion of linear chromosomes. However, telomerase could promote telomere lengthening and telomere length homeostasis in stem cells and malignant cells [15-17]. Multiple studies indicate that telomere length in peripheral blood lymphocytes was significant associated with cancer susceptibility [18-23]. Either extreme short leukocyte telomere length or extreme long leukocyte telomere length may contribute to cancer development. Genomic instability, a hallmark of cancer, occurs in cells with shorten telomeres and, thus, individuals with extreme short leukocyte telomeres may confer to elevated risk of several cancers such as ESCC $[17,24,25]$.

The heritability estimates of human telomere length are $44 \% \sim 80 \%$, suggesting the key role of genetic factors in controlling telomere length $[26,27]$. Several quantitative trait linkage (QTL) GWAS and candidate gene QTL studies have mapped various SNPs correlated to leucocyte telomere length [28-37]. Several telomere length-related SNPs have been previously found to confer susceptibility of cancers including ESCC. For instance, we previously identified two SNPs (rs621559 and rs398652) which are significantly associated with telomere length in Chinese as well as ESCC predisposition [24]. However, it is still largely unclear how recent GWAS identified telomere length-related SNPs impact ESCC development. Following these leads we performed an in-depth analysis of the genetic variability of these telomere length-related SNPs in ESCC.

\section{Material and Methods}

\section{Study case-control sets}

In the current study, there are two case-control sets, Jiangsu discovery set and Shandong validation set (Supplementary Table 1). The detailed information on subject recruitments has been reported in details previously [38]. All subjects enrolled in the study were ethnic Han Chinese. This study was approved by the Institutional Review Boards. At recruitment, the written informed consent was obtained from each subject. The methods were carried out in accordance with the approved guidelines.

\section{Measurement of leukocyte telomere length}

As reported previously, telomere length of leukocytes was detected using quantitative real-time
PCR [39]. All quantitative real-time PCR reactions were performed on ABI QuantStudio 6 Flex RealTime PCR System (Foster City, CA, US). The relative leukocyte telomere lengths were calculated as the ratio of the telomere repeat copy number and the $\beta$-globin gene copy number (the T/S ratio).

\section{SNPs selection and genotyping}

In a previous study, we identified seven SNPs (CXCR4 rs6430612, TERT rs13172201, TERT rs10069690, TERT rs2853676, TERT rs451360, OBFC1 rs4387287, and VPS34 rs2162440) are significantly or marginally associated with telomere length of leucocytes in Shandong heathy subjects (all $P<0.10$ ). [40].In this study, these SNPs were firstly genotyped in the controls of Jiangsu discovery set using the iPLEX Sequenom MassARRAY platform (Sequenom Inc., San Diego, CA, USA) as reported previously [40]. Telomere length-related CXCR4 rs6430612, TERT rs13172201, and OBFC1 rs4387287 SNPs were then analyzed in both discovery and validation casecontrol sets. A $15 \%$ random sample was reciprocally tested and the reproducibility was $100 \%$.

\section{Statistics}

The differences in demographic variables, smoking status, drinking status between patients and controls were examined using Pearson's $X^{2}$ test. The association between relative telomere lengths of leukocytes and the SNPs were calculated using multivariable linear regression adjusted for age and sex. The associations of CXCR4 rs6430612, TERT rs13172201, and OBFC1 rs4387287 with ESCC risk were estimated by odds ratios (ORs) and their 95\% confidence intervals ( $95 \%$ CIs) computed by logistic regression models. All ORs were adjusted for age, sex, smoking or drinking status, where it was appropriate. A $P$ value of less than 0.05 was used as the criterion of statistical significance. Bonferroni correction was used for multiple comparisons. All statistical tests were two-sided. All analyses were performed using SPSS 16.0 (SPSS Inc.).

\section{Results}

\section{Identification of SNPs significantly associated with telomere length}

Our previous study indicated that CXCR4 rs6430612, TERT rs13172201, TERT rs10069690, TERT rs2853676, TERT rs451360, OBFC1 rs4387287, and VPS34 rs2162440 are significantly or marginally associated with telomere length of leucocytes. As a result, we firstly validated the association of these genetic polymorphisms with telomere length in heathy subjects of Jiangsu set. As shown in Table 1, we observed significant correlations between CXCR4 
rs6430612, TERT rs13172201, and OBFC1 rs4387287 SNPs and telomere length (all $P<0.05$ ). In details, the minor alleles of CXCR4 rs6430612 and OBFC1 rs4387287 genetic variations are remarkably associated with long telomere length. On the contrary, the TERT rs13172201 $\mathrm{T}$ allele is associated with markedly short leukocyte telomere and showed to be risk allele. However, we did not find that TERT rs10069690, TERT rs2853676, TERT rs451360 and OBFC1 rs4387287 polymorphisms are significantly associated with telomere length of leukocytes in Jiangsu set (all $P>0.05)$ (Table 1 ).

\section{Association between rs6430612, rs13172201 and rs4387287 SNPs and ESCC risk}

To explore the impact of telomere length-related CXCR4 rs6430612, TERT rs13172201 and OBFC1 rs4387287 polymorphisms on ESCC susceptibility, we next genotyped the SNPs in both Jiangsu and Shandong sets. Unconditional logistic regression analyses showed that the odds of having the CXCR4 rs6430612 CT genotype in ESCC patients was 0.40 (95\% CI $\left.=0.25-0.62, P=4.7 \times 10^{-5}\right)$ compared with carriers of the CC genotype in the Jiangsu set (Table 2). Importantly, individuals with the CXCR4 rs6430612 CT genotype showed $43 \%$ decreased risk to develop ESCC compared with those with the CXCR4 CC genotype in Shandong set $(95 \% \mathrm{CI}=0.42-0.78, P=$ $4.0 \times 10^{-4}$ ) (Table 2). Pooled analyses indicated that the odds of having the CXCR4 rs6430612 CT genotype in ESCC patients was $0.51(95 \% \mathrm{CI}=0.40-0.66, P=$ $1.7 \times 10^{-7}$, still statistically significant after Bonferroni corrections) compared to the CC genotype (Table 2).

Logistic regression analyses elucidated that the TERT rs13172201 T allele was ESCC risk allele. Individuals carrying the CT or TT genotype had an OR of $1.42(95 \% \mathrm{CI}=1.08-1.88, P=0.012)$ or $1.44(95 \%$ $\mathrm{CI}=0.93-2.21, P=0.102)$ for developing ESCC, respectively, compared with individuals having the CC genotype in the discovery set (Table 3 ). In line with this, carriers of TERT rs13172201 CT or TT genotype showed 1.33-fold elevated risk to develop
ESCC compared with carriers of the CC genotype (95\% CI $=1.08-1.64, P=0.007 ; \mathrm{OR}=1.52,95 \% \mathrm{CI}=$ 1.14-2.02, $P=0.004$ ) in Shandong set (Table 3). Pooled analyses all support the hazardous role of the TERT rs13172201 T allele in ESCC (CT genotype: OR $=1.37$, $95 \% \mathrm{CI}=1.16-1.61, P=2.2 \times 10^{-4}$; $\mathrm{TT}$ genotype: $\mathrm{OR}=$ $1.50,95 \% \mathrm{CI}=1.19-1.91, P=0.001$, still statistically significant after Bonferroni corrections) (Table 3).

In Jiangsu set, a 0.74-fold reduced risk of developing ESCC was associated with the OBFC1 rs4387287 CA genotype (95\% CI $=0.55-0.99 ; P=0.049$ ) compared with the $\mathrm{CC}$ genotype. Consistently, there was a 0.67 -fold $\left(95 \% \mathrm{CI}=0.54-0.84, P=3.9 \times 10^{-4}\right)$ or 0.64 -fold $(95 \% \mathrm{CI}=0.44-0.95, P=0.025)$ decreased ESCC risk was observed among carriers of the OBFC1 rs4387287 CA or AA genotype compared to individuals with the CC genotype in Shandong set (Table 4). In the pooled analyses, similar results were observed (the CA genotype: $\mathrm{OR}=0.67,95 \% \mathrm{CI}=$ $0.58-0.82, P=3.9 \times 10^{-5}$; the AA genotype: $\mathrm{OR}=0.59$, $95 \% \mathrm{CI}=0.43-0.82, P=0.002$, still statistically significant after Bonferroni corrections) (Table 4).

\section{Stratified analyses of association between rs6430612, rs13172201 and rs4387287 SNPs and ESCC risk}

ESCC genetic susceptibility associated with telomere length-related CXCR4 rs6430612, TERT rs13172201 and OBFC1 rs4387287 polymorphisms was further investigated by stratifying for age, sex, smoking and alcohol drinking status using pooled data of two case-control sets (Table 5). For the CXCR4 rs6430612 polymorphism, a decreased risk of ESCC was associated with the CT or TT genotype in both individuals aged older than 66 years and ones aged 66 years or younger (both $P<0.05$ ). Similarly, in stratified analyses with sex, smoking or alcohol drinking status, the CXCR4 rs6430612 genetic variant was significantly associated with reduced risk in males, females, smokers, nonsmokers, drinkers or nondrinkers (all $P<0.05$ ) (Table 5).

Table 1. Association between telomere length-related genetic variations from previously published studies with leucocyte telomere length in heathy subjects of Jiangsu discovery set

\begin{tabular}{|c|c|c|c|c|c|c|c|}
\hline Genes & SNP IDs & Location $^{1}$ & Alleles & $\beta$-value ${ }^{1}$ & SE & $P^{2}$ & Reference \\
\hline CXCR4 & rs6430612 & Chromosome 2:137006198 & $\mathrm{C} / \mathrm{T}$ & -0.112 & 0.061 & 0.044 & Levy D, et al., 2010 \\
\hline TERT & rs13172201 & Chromosome 5:1271661 & $\mathrm{C} / \mathrm{T}$ & 0.125 & 0.056 & 0.022 & Bao Y, et al., 2017 \\
\hline TERT & rs10069690 & Chromosome 5:1279790 & $\mathrm{C} / \mathrm{T}$ & -0.069 & 0.063 & 0.183 & Julin B, et al., 2015 \\
\hline TERT & rs2853676 & Chromosome 5:1288547 & $\mathrm{C} / \mathrm{T}$ & -0.023 & 0.053 & 0.668 & Julin B, et al., 2015 \\
\hline TERT & rs451360 & Chromosome 5:1319680 & $\mathrm{C} / \mathrm{A}$ & 0.143 & 0.047 & 0.036 & Bao Y, et al., 2017 \\
\hline OBFC1 & rs4387287 & Chromosome 10:105677897 & $\mathrm{A} / \mathrm{C}$ & -0.134 & 0.051 & 0.011 & Levy D, et al., 2010 \\
\hline VPS34 & rs2162440 & Chromosome 18:35214006 & $\mathrm{G} / \mathrm{A}$ & 0.013 & 0.029 & 0.782 & Mangino M, et al., 2009 \\
\hline
\end{tabular}

Note: SNP, single nucleotide polymorphism

1: Reference genome GRCh37.p13.

2: The association of SNPs with telomere length was assessed using linear regression adjusted for age sex, smoking and drinking status. 
Table 2. Genotype frequencies of CXCR4 rs6430612 genetic variant among patients and controls and their association with ESCC risk

\begin{tabular}{llllll}
\hline & Genotypes & $\begin{array}{l}\text { Patients No. } \\
(\%)\end{array}$ & $\begin{array}{l}\text { Controls } \\
\text { No. }(\%)\end{array}$ & OR $^{1}(95 \%$ CI $)$ & $P$-value \\
\hline Jiangsu & & $n=588$ & $n=600$ & & \\
set & CC & $549(93.4)$ & $517(86.2)$ & Reference & \\
& CT & $37(6.3)$ & $78(13.0)$ & $0.40(0.25-0.62)$ & $4.7 \times 10^{-5}$ \\
& TT & $2(0.3)$ & $5(0.8)$ & N.C. & N.C. \\
Shandong & & $n=997$ & $n=1000$ & & \\
set & CC & $911(91.4)$ & $850(85.0)$ & Reference & \\
& CT & $84(8.4)$ & $139(13.9)$ & $0.57(0.42-0.78)$ & $4.0 \times 10^{-4}$ \\
& TT & $2(0.2)$ & $11(1.1)$ & N.C. & N.C. \\
Total & & $n=1585$ & $n=1600$ & & \\
& CC & $1460(92.1)$ & $1367(85.4)$ & Reference & \\
& CT & $121(7.6)$ & $217(13.6)$ & $0.51(0.40-0.66)$ & $1.7 \times 10^{-7}$ \\
& TT & $4(0.3)$ & $16(1.0)$ & N.C. & N.C. \\
\hline
\end{tabular}

Abbreviations: ESCC, esophageal squamous cell carcinoma; OR, odds ratio; CI, confidence interval; N.C., not calculated.

1: Data were calculated by logistic regression with adjustment for age, sex, smoking and drinking status.

Table 3. Genotype frequencies of TERT rs13172201 genetic variant among patients and controls and their association with ESCC risk

\begin{tabular}{|c|c|c|c|c|c|}
\hline & Genotypes & $\begin{array}{l}\text { Patients } \\
\text { No. }(\%)\end{array}$ & $\begin{array}{l}\text { Controls } \\
\text { No. (\%) }\end{array}$ & $\mathrm{OR}^{1}(95 \% \mathrm{CI})$ & $P$-value \\
\hline \multirow[t]{4}{*}{ Jiangsu set } & & $n=588$ & $n=600$ & & \\
\hline & CC & $367(62.4)$ & $432(72.0)$ & Reference & \\
\hline & $\mathrm{CT}$ & 199(33.8) & $158(26.3)$ & $1.42(1.08-1.88)$ & 0.012 \\
\hline & TT & $22(3.7)$ & $10(1.7)$ & $1.44(0.93-2.21)$ & 0.102 \\
\hline \multirow{4}{*}{$\begin{array}{l}\text { Shandong } \\
\text { set }\end{array}$} & & $n=1000$ & $n=1000$ & & \\
\hline & $\mathrm{CC}$ & $623(62.3)$ & $713(71.3)$ & Reference & \\
\hline & $\mathrm{CT}$ & $329(32.9)$ & $267(26.7)$ & $1.33(1.08-1.64)$ & 0.007 \\
\hline & TT & $48(4.8)$ & $20(2.0)$ & $1.52(1.14-2.02)$ & 0.004 \\
\hline \multirow[t]{4}{*}{ Total } & & $n=1588$ & $n=1600$ & & \\
\hline & $\mathrm{CC}$ & $990(62.3)$ & 1145(71.6) & Reference & \\
\hline & $\mathrm{CT}$ & $528(33.2)$ & $425(26.6)$ & $1.37(1.16-1.61)$ & $2.2 \times 10^{-4}$ \\
\hline & TT & $70(4.4)$ & $30(1.9)$ & $1.50(1.19-1.91)$ & 0.001 \\
\hline
\end{tabular}

Abbreviations: ESCC, esophageal squamous cell carcinoma; OR, odds ratio; CI, confidence interval.

1: Data were calculated by logistic regression with adjustment for age, sex, smoking and drinking status.

Table 4. Genotype frequencies of OBFCl rs4387287 genetic variant among patients and controls and their association with ESCC risk

\begin{tabular}{llllll}
\hline & \multicolumn{2}{c}{ Genotypes $\begin{array}{l}\text { Patients } \\
\text { No. }(\%)\end{array}$} & $\begin{array}{l}\text { Controls No. } \\
(\%)\end{array}$ & OR $^{1}(95 \%$ CI $)$ & $P$-value \\
\hline Jiangsu set & & $n=588$ & $n=600$ & & \\
& CC & $469(79.8)$ & $422(70.4)$ & Reference & \\
& CA & $115(19.5)$ & $164(27.3)$ & $0.74(0.55-0.99)$ & 0.049 \\
& AA & $4(0.7)$ & $14(2.3)$ & N.C. & N.C. \\
Shandong & & $n=1000$ & $n=1000$ & & \\
set & CC & $776(77.6)$ & $707(70.7)$ & Reference & \\
& CA & $213(21.3)$ & $268(26.8)$ & $0.67(0.54-0.84)$ & $3.9 \times 10^{-4}$ \\
Total & AA & $11(1.1)$ & $25(2.5)$ & $0.64(0.44-0.95)$ & 0.025 \\
& & $n=1588$ & $n=1600$ & & \\
& CC & $1245(78.4)$ & $1129(70.6)$ & Reference & \\
& CA & $328(20.7)$ & $432(27.0)$ & $0.67(0.58-0.82)$ & $3.9 \times 10^{-5}$ \\
& AA & $15(0.9)$ & $39(2.4)$ & $0.59(0.43-0.82)$ & 0.002
\end{tabular}

Abbreviations: ESCC, esophageal squamous cell carcinoma; OR, odds ratio; CI, confidence interval; N.C., not calculated.

1 : Data were calculated by logistic regression with adjustment for age, sex, smoking and drinking status.
For the TERT rs13172201 SNP, a significantly elevated risk of ESCC associated with the CT or TT genotype was observed among nonsmokers $(\mathrm{OR}=$ $\left.1.82,95 \% \mathrm{CI}=1.41-2.33, P=2.8 \times 10^{-6}\right)$, but not among smokers $(\mathrm{OR}=1.20,95 \% \mathrm{CI}=0.97-1.49, P=0.090)$. There was statistically significant gene-smoking interaction for the TERT rs13172201 SNP $\left(P_{\text {interaction }}=\right.$ $0.024)$. In stratified analyses with age, sex or alcohol drinking status, the TERT rs13172201 polymorphism was markedly associated with increased risk in all sub-groups (all $P<0.05$ ). No significant geneenvironment interactions were observed (Table 5, Table 6).

For the OBFC1 rs4387287 polymorphism, stratified analyses showed that significantly decreased ORs for ESCC development were only observed in males $(\mathrm{OR}=0.57,95 \% \mathrm{CI}=0.47-0.70, P=$ $\left.2.3 \times 10^{-7}\right)$, smokers $(\mathrm{OR}=0.44,95 \% \mathrm{CI}=0.35-0.55, P=$ $\left.1.5 \times 10^{-7}\right)$, and alcohol drinkers $(\mathrm{OR}=0.40,95 \% \mathrm{CI}=$ $\left.0.32-0.52, P=7.6 \times 10^{-8}\right)$. A statistically significant gene-sex, gene-smoking or gene-drinking interaction was observed $\left(P_{\text {interaction }}=0.001,0.002\right.$ or $\left.2.2 \times 10^{-4}\right)$. However, there was no significantly decreased risk for females, nonsmokers or nondrinkers with the OBFC1 rs4387287 CA or AA genotype compared with ones with the CC genotype. Evident association between the rs4387287 CA or AA genotype and ESCC susceptibility was found both individuals aged older than 66 years and ones aged 66 years or younger (both $P<0.05$ ) (Table 5, Table 6).

\section{Discussion}

Due to incomplete replication of the 3 ' end of each DNA strand, gradual shrink of telomeres after every mitotic division would lead to chromosomal instability of somatic cells. Genome instability caused by shorten telomere length will finally promote oncogenic phenotypes and malignant transformation of normal cells. Considering short telomere length of leukocytes has been associated with increased ESCC susceptibilities [24,25], we hypothesized that germline genetic variants associated with shortened telomeres may contribute to increased ESCC risk. To test this notion, in the present study, we systematically evaluate thirty candidate genetic variants reported previously and firstly identified three SNPs associated with telomere length in Chinese. Based on analyzing 1,588 ESCC cases and 1,600 controls, we found that CXCR4 rs6430612 and OBFC1 rs4387287 polymorphisms significantly confer reduced risk of ESCC. On the contrary, an evidently increased OR for ESCC development was associated with TERT rs13172201 genetic variant.

We previously found that CXCR4 rs6430612 polymorphism is a telomere length-related SNP 
contributing to GCA risk, with the $\mathrm{T}$ allele as a protective allele [40].CXCR4 is a chemokine receptor whose expression was significantly correlated with invasion, angiogenesis, metastasis, and prognosis of ESCC [41-43]. For instance, expression of CXCR4 in ESCC is of major relevance in a German population [41]. High expression levels of CXCR4 in cytoplasm and nuclei were associated with poor cause-specific survival in Japanese [43]. In a Chinese population, the expression of CXCR4 in tumor cells was positively associated with tumor status and clinical stage [42]. In addition, ESCC cells coexpressing CD133 and CXCR4 possess the characteristics of cancer stem cells and contribute to poor prognosis of patients [44]. The crucial involvement of CXCR4 in ESCC development may illuminates the significant association between the rs6430612 polymorphism and ESCC risk.

The TERT gene locating at chromosome 5p15.33 encodes the catalytic subunit of telomerase reverse transcriptase. TERT is a key component of the RNAprotein complex which functions in maintaining telomere ends. Accumulated evidences indicate that TERT SNPs contribute to ESCC risk [45-47]. For the rs13172201 polymorphism, Wang et al identified it as one of five TERT independent risk loci across different cancer types $\left(P=0.041\right.$ and $\left.P_{\text {Conditional }}=2.04 \times 10^{-6}\right)$ based on sequential conditional analyses [48]. Consistent with this, we demonstrated that a significantly elevated ESCC risk was associated with minor alleles of the rs13172201 variant.

The OBFC1 gene, also known as STN1, codes a protein which is a subunit of a telomere-associated complex including C17ORF68 and TEN1 [49]. OBFC1 is also one of the components of an alpha accessory factor that promotes the activity of DNA polymerasealpha-primase, the enzyme that initiates DNA replication. Importantly, human OBFC1 locus genetic variants are involved in telomere biology [50] and confer risk of melanoma, epithelial ovarian cancer, thyroid cancer, uterine leiomyoma and pancreatic cancer [51-56]. However, several cancer susceptibility OBFC1 polymorphisms in Caucasian populations, such as rs7902587, rs2487999 and rs9420907, do not exist in Han Chinese populations except rs4387287. Strikingly, we found that the OBFC1 rs4387287 variant significantly contributes to ESCC risk. In the stratified analyses, we observed a significant gene-smoking or gene-drinking interaction for rs4387287. The exact mechanisms for these gene-environment interactions are currently unknown. However, a systematic review of 84 studies and meta-analysis indicated that shorter telomeres were found among ever smokers compared to those who never smoked [57], which may contribute at least in part to our stratified results. Together, these results add new lines of evidences highlighting the role of $O B F C 1$ in telomere-related malignant transformation.

There are several limitations in the current study. First of all, inherent selection bias may exist since this hospital-based study enrolled ESCC cases and healthy controls from hospitals. Therefore, it is important to validate these findings in a populationbased prospective study. Second, the sample size of this study may limit the statistical power for statistical analyses of gene-covariate interactions. Third, future studies will need to address these polymorphisms with other exposure risk factors of ESCC such as nutritional deficiencies and nitrosamines.

In summary, we found that CXCR4 rs6430612, TERT rs13172201 and OBFC1 rs4387287 are key genetic components in complicated regulation of telomere length. In accord with this notion, these polymorphisms also significantly contribute to susceptibility to ESCC. Our results elucidate the prevalent involvement of genetic variants in telomere biology and further provide pathogenic insights into the role of telomere in cancer development.

Table 5. Risk of ESCC associated with CXCR4, TERT and OBFCI SNPs by age, sex, smoking, and drinking status

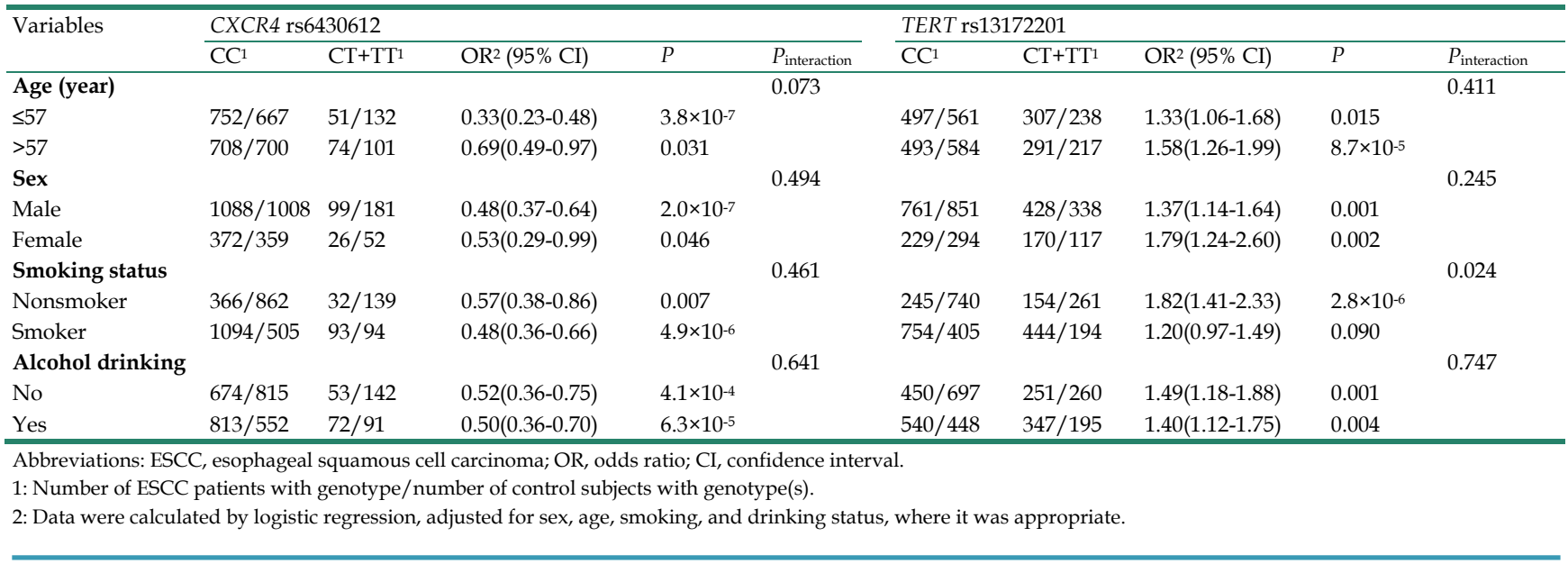


Table 6. Risk of ESCC associated with CXCR4, TERT and VPS34 SNPs by age, sex, smoking, and drinking status (continued)

\begin{tabular}{|c|c|c|c|c|c|}
\hline \multirow[t]{2}{*}{ Variables } & \multicolumn{5}{|c|}{ OBFC1 rs4387287 } \\
\hline & $\mathrm{CC}^{1}$ & $\mathrm{CA}+\mathrm{AA}^{1}$ & $\mathrm{OR}^{2}(95 \% \mathrm{CI})$ & $P$ & $P_{\text {interaction }}$ \\
\hline Age (year) & & & & & 0.969 \\
\hline$\leq 57$ & $623 / 563$ & $181 / 236$ & $0.64(0.50-0.82)$ & $4.8 \times 10^{-4}$ & \\
\hline$>57$ & $622 / 566$ & $162 / 235$ & $0.63(0.50-0.81)$ & $3.1 \times 10^{-4}$ & \\
\hline Sex & & & & & 0.001 \\
\hline Male & $946 / 820$ & $243 / 329$ & $0.57(0.47-0.70)$ & $2.3 \times 10^{-7}$ & \\
\hline Female & $299 / 309$ & $100 / 102$ & $1.08(0.72-1.62)$ & 0.723 & \\
\hline Smoking status & & & & & 0.002 \\
\hline Nonsmoker & $285 / 737$ & $114 / 264$ & $1.09(0.84-1.42)$ & 0.503 & \\
\hline Smoker & $960 / 392$ & $229 / 207$ & $0.44(0.35-0.55)$ & $1.5 \times 10^{-7}$ & \\
\hline Alcohol drinking & & & & & $2.2 \times 10^{-4}$ \\
\hline No & $512 / 706$ & $189 / 251$ & $1.06(0.83-1.35)$ & 0.661 & \\
\hline Yes & $733 / 423$ & $154 / 220$ & $0.40(0.32-0.52)$ & $7.6 \times 10^{-8}$ & \\
\hline
\end{tabular}

Abbreviations: ESCC, esophageal squamous cell carcinoma; OR, odds ratio; CI, confidence interval.

1: Number of ESCC patients with genotype/number of control subjects with genotype(s).

2: Data were calculated by logistic regression, adjusted for sex, age, smoking, and drinking status, where it was appropriate.

\section{Supplementary Material}

Supplementary table S1.

http://www.jcancer.org/v11p5025s1.pdf

\section{Acknowledgements}

This study was financially supported by National Natural Science Foundation of China (31671300, 31871306); Taishan Scholars Program of Shandong Province (tsqn20161060); Program of Science and Technology for the youth innovation team in universities of Shandong Province (2020KJL001); the Academic Promotion Program of Shandong First Medical University (2019RC001).

\section{Competing Interests}

The authors have declared that no competing interest exists.

\section{References}

1. Bray F, Ferlay J, Soerjomataram I, et al. Global cancer statistics 2018: GLOBOCAN estimates of incidence and mortality worldwide for 36 cancers in 185 countries. CA Cancer J Clin. 2018; 6: 394-424.

2. Tran GD, Sun $X D$, Abnet CC, et al. Prospective study of risk factors for esophageal and gastric cancers in the Linxian general population trial cohort in China. Int J Cancer. 2005; 113: 456-63.

3. Islami F, Kamangar F, Aghchel K, et al. Epidemiologic features of upper gastrointestinal tract cancers in Northeastern Iran. Br J Cancer. 2004; 90: $1402-6$

4. Wu M, Liu AM, Kampman E, et al. Green tea drinking, high tea temperature and esophageal cancer in high- and low-risk areas of Jiangsu Province, China: a population-based case-control study. Int J Cancer. 2009; 124: 1907-13.

5. Chang J, Zhong R, Tian J, et al. Exome-wide analyses identify low-frequency variant in CYP26B1 and additional coding variants associated with esophageal squamous cell carcinoma. Nat Genet. 2018; 50: 338-43.

6. Wu C, Wang Z, Song X, et al. Joint analysis of three genome-wide association studies of esophageal squamous cell carcinoma in Chinese populations. Nat Genet. 2014; 46: 1001-6.

7. Yan C, Ji Y, Huang T, et al. An esophageal adenocarcinoma susceptibility locus at $9 q 22$ also confers risk to esophageal squamous cell carcinoma by regulating the function of BARX1. Cancer Lett. 2018; 421: 103-11.

8. Wu C, Kraft P, Zhai K, et al. Genome-wide association analyses of esophageal squamous cell carcinoma in Chinese identify multiple susceptibility loci and gene-environment interactions. Nat Genet. 2012; 44: 1090-7.

9. Abnet CC, Wang Z, Song X, et al. Genotypic variants at $2 \mathrm{q} 33$ and risk of esophageal squamous cell carcinoma in China: a meta-analysis of genome-wide association studies. Hum Mol Genet. 2012; 21: 2132-41.
10. Wu C, Hu Z, He Z, et al. Genome-wide association study identifies three new susceptibility loci for esophageal squamous-cell carcinoma in Chinese populations. Nat Genet. 2011; 43: 679-84.

11. Tanaka F, Yamamoto K, Suzuki S, et al. Strong interaction between the effects of alcohol consumption and smoking on oesophageal squamous cell carcinoma among individuals with ADH1B and/or ALDH2 risk alleles. Gut. 2010; 59: 1457-64.

12. Wang LD, Zhou FY, Li XM, et al. Genome-wide association study of esophageal squamous cell carcinoma in Chinese subjects identifies susceptibility loci at PLCE1 and C20orf54. Nat Genet. 2010; 42: 759-63.

13. Abnet CC, Freedman ND, Hu N, et al. A shared susceptibility locus in PLCE1 at 10q23 for gastric adenocarcinoma and esophageal squamous cell carcinoma. Nat Genet. 2010; 42: 764-7.

14. Cui R, Kamatani Y, Takahashi A, et al. Functional variants in ADH1B and ALDH2 coupled with alcohol and smoking synergistically enhance esophageal cancer risk. Gastroenterology. 2009; 137: 1768-75.

15. Shay JW. Telomeres and aging. Curr Opin Cell Biol. 2018; 52: 1-7.

16. Tomita K. How long does telomerase extend telomeres? Regulation of telomerase release and telomere length homeostasis. Curr Genet. 2018; 64: 1177-81.

17. Aviv A, Anderson JJ, Shay JW. Mutations, Cancer and the Telomere Length Paradox. Trends Cancer. 2017; 3: 253-8.

18. Zhang $\mathrm{X}$, Zhao $\mathrm{Q}$, Zhu $\mathrm{W}$, et al. The Association of Telomere Length in Peripheral Blood Cells with Cancer Risk: A Systematic Review and Meta-analysis of Prospective Studies. Cancer Epidemiol Biomarkers Prev. 2017; 26: 1381-90.

19. Martinez P, Blasco MA. Telomere-driven diseases and telomere-targeting therapies. J Cell Biol. 2017; 216: 875-87.

20. Jafri MA, Ansari SA, Alqahtani MH, Shay JW. Roles of telomeres and telomerase in cancer, and advances in telomerase-targeted therapies. Genome Med. Genome Med. 2016; 8: 69.

21. Sha JW. Role of Telomeres and Telomerase in Aging and Cancer. Cancer Discov. 2016; 6: 584-93.

22. Sarek G, Marzec P, Margalef P, Boulton SJ. Molecular basis of telomere dysfunction in human genetic diseases. Nat Struct Mol Biol. 2015; 22: 867-74.

23. Li J, An C, Zheng $\mathrm{H}$, et al. Leukocyte Telomere Length and Risk of Papillary Thyroid Carcinoma. J Clin Endocrinol Metab. 2019; 104: 2712-8.

24. Shi J, Sun F, Peng L, et al. Leukocyte telomere length-related genetic variants in 1p34.2 and 14q21 loci contribute to the risk of esophageal squamous cell carcinoma. Int J Cancer. 2013; 132: 2799-807.

25. Pan W, Du J, Shi M, Jin G, Yang M. Short leukocyte telomere length, alone and in combination with smoking, contributes to increased risk of gastric cancer or esophageal squamous cell carcinoma. Carcinogenesis. 2017; 38: 12-8.

26. Vasa-Nicotera M, Brouilette $S$, Mangino M, et al. Mapping of a major locus that determines telomere length in humans. Am J Hum Genet. 2005; 76: 147-51.

27. Njajou OT, Cawthon RM, Damcott CM, et al. Telomere length is paternally inherited and is associated with parental lifespan. Proc Natl Acad Sci U S A. 2007; 104: 12135-9.

28. Ojha J, Codd V, Nelson CP, et al. Genetic Variation Associated with Longer Telomere Length Increases Risk of Chronic Lymphocytic Leukemia. Cancer Epidemiol Biomarkers Prev. 2016; 25: 1043-9.

29. Julin B, Shui I, Heaphy CM, et al. Circulating leukocyte telomere length and risk of overall and aggressive prostate cancer. Br J Cancer. 2015; 112: 769-76.

30. Levy D, Neuhausen SL, Hunt SC, et al. Genome-wide association identifies OBFC1 as a locus involved in human leukocyte telomere biology. Proc Natl Acad Sci U S A. 2010; 107: 9293-8.

31. Shen $\mathrm{Q}$, Zhang $\mathrm{Z}$, Yu L, et al. Common variants near TERC are associated with leukocyte telomere length in the Chinese Han population. Eur J Hum Genet. 2011; 19: 721-3 
32. Jones AM, Beggs AD, Carvajal-Carmona L, et al. TERC polymorphisms are associated both with susceptibility to colorectal cancer and with longer telomeres. Gut. 2012; 61: 248-54.

33. Terry KL, Tworoger SS, Vitonis AF, et al. Telomere length and genetic variation in telomere maintenance genes in relation to ovarian cancer risk. Cancer Epidemiol Biomarkers Prev. 2012; 21: 504-12.

34. Codd V, Mangino M, van der Harst $\mathrm{P}$, et al. Common variants near TERC are associated with mean telomere length. Nat Genet. 2010; 42: 197-9.

35. Mangino M, Richards JB, Soranzo N, et al. A genome-wide association study identifies a novel locus on chromosome 18q12.2 influencing white cell telomere length. J Med Genet. 2009; 46: 451-4.

36. Concetti F, Carpi FM, Nabissi M, Picciolini M, Santoni G, Napolioni V. The functional polymorphism rs73598374:G>A (p.Asp8Asn) of the ADA gene is associated with telomerase activity and leukocyte telomere length. Eur J Hum Genet. 2015; 23: 267-70.

37. Walsh KM, Codd V, Smirnov IV, et al. Variants near TERT and TERC influencing telomere length are associated with high-grade glioma risk. Nat Genet. 2014; 46: 731-5.

38. Shi M, Xia J, Xing H, et al. The Sp1-mediaded allelic regulation of MMP13 expression by an ESCC susceptibility SNP rs2252070. Sci Rep. 2016; 6: 27013.

39. Cawthon RM. Telomere measurement by quantitative PCR. Nucleic Acids Res. 2002; 30: e47.

40. Zhang N, Zheng Y, Liu J, Lei T, Xu Y, Yang M. Genetic variations associated with telomere length confer risk of gastric cardia adenocarcinoma. Gastric Cancer. 2019; 22: 1089-99.

41. Gockel I, Schimanski CC, Heinrich C, et al. Expression of chemokine receptor CXCR4 in esophageal squamous cell and adenocarcinoma. BMC Cancer. 2006; 6: 290.

42. Zhang L, Ye SB, Ma G, et al. The expressions of MIF and CXCR4 protein in tumor microenvironment are adverse prognostic factors in patients with esophageal squamous cell carcinoma. J Transl Med. 2013; 11: 60.

43. Goto M, Yoshida T, Yamamoto Y, et al. CXCR4 Expression is Associated with Poor Prognosis in Patients with Esophageal Squamous Cell Carcinoma. Ann Surg Oncol. 2017; 24: 832-40.

44. $\mathrm{Lu} \mathrm{C}, \mathrm{Xu} \mathrm{F}, \mathrm{Gu} \mathrm{J}$, et al. Clinical and biological significance of stem-like CD133(+) CXCR4(+) cells in esophageal squamous cell carcinoma. J Thorac Cardiovasc Surg. 2015; 150: 386-95.

45. Zhou L, Fu G, Wei J, et al. The identification of two regulatory ESCC susceptibility genetic variants in the TERT-CLPTM1L loci. Oncotarget. 2016; 7: 5495-506.

46. Du J, Xue W, Ji Y, et al. U-shaped association between telomere length and esophageal squamous cell carcinoma risk: a case-control study in Chinese population. Front Med. 2015; 9: 478-86.

47. Yin J, Wang L, Zheng L, et al. TERT-CLPTM1L Rs401681 C>T polymorphism was associated with a decreased risk of esophageal cancer in a Chinese population. PLoS One. 2014; 9: e100667.

48. Wang Z, Zhu B, Zhang M, et al. Imputation and subset-based association analysis across different cancer types identifies multiple independent risk loci in the TERT-CLPTM1L region on chromosome 5p15.33. Hum Mol Genet. 2014; 23: 6616-33.

49. Miyake Y, Nakamura M, Nabetani A, et al. RPA-like mammalian Ctc1-Stn1-Ten1 complex binds to single-stranded DNA and protects telomeres independently of the Pot1 pathway. Mol Cell. 2009; 36: 193-206.

50. Rode L, Nordestgaard BG, Bojesen SE. Peripheral blood leukocyte telomere length and mortality among 64,637 individuals from the general population. J Natl Cancer Inst. J Natl Cancer Inst. 2015; 107: djv074.

51. Phelan $\mathrm{CM}, \mathrm{Kuchenbaecker} \mathrm{KB}$, Tyrer JP, et al. Identification of 12 new susceptibility loci for different histotypes of epithelial ovarian cancer. Nat Genet. 2017; 49: 680-91.

52. Duffy DL, Zhu G, Li X, et al. Novel pleiotropic risk loci for melanoma and nevus density implicate multiple biological pathways. Nat Commun. 2018; 9: 4774 .

53. Gudmundsson J, Thorleifsson G, Sigurdsson JK, et al. A genome-wide association study yields five novel thyroid cancer risk loci. Nat Commun. 2017; 8: 14517.

54. Valimaki N, Kuisma H, Pasanen A, et al. Genetic predisposition to uterine leiomyoma is determined by loci for genitourinary development and genome stability. Elife. 2018; 7

55. Rafnar T, Gunnarsson B, Stefansson OA, et al. Variants associating with uterine leiomyoma highlight genetic background shared by various cancers and hormone-related traits. Nat Commun. 2018; 9: 3636.

56. Campa D, Matarazzi M, Greenhal W, et al. Genetic determinants of telomere length and risk of pancreatic cancer: A PANDoRA study. Int J Cancer. 2019; 144: $1275-83$

57. Astuti Y, Wardhana A, Watkins J, et al. Cigarette smoking and telomere length: A systematic review of 84 studies and meta-analysis. Environ Res. 2017;158:480-9. 